Z. Klin. Chem. Klin. Biochem.

12. Jg. 1974 , S. $361-366$

\title{
Über den Einfluß von Fett auf die Proteinbestimmung mit Biuret- und Folin-Ciocalteus Phenol-Rea- genz in Leberhomogenaten
}

\author{
Von $A$. Metz und K. Reinert \\ Medizinisch-Biologische Forschungsabteilung Sandoz AG, Basel (Schweiz)
}

(Eingegangen am 8. Februar/29. Mai 1974)

Die vorliegende Studie behandelt die Problematik der photometrischen Proteinbestimmung in Leberhomogenaten in Anwesenheit steigender Fettmengen und zeigt die dabei auftretenden Fehlermöglichkeiten auf. Es wird vorgeschlagen, dicse Fettrübungseinflüsse entweder durch Detergenzzusatz (Desoxycholat) oder durch Verwendung von Spektralphotometern mit speziellen Meßanordnungen für Trübungsmessungen zu unterdrücken.

\section{The influence of fat on the determination of protein by the biuret and the Folin-Ciocalteau methods in liver homogenates}

The present study deals with the spectrophotometric determination of protein in liver homogenates and the errors of determination liable to occur due to clouding of the test sample by the presence of lipids. Two ways of suppressing this source of error are described: the addition of a detergent (desoxycholate) to the test sample to clear the clouding, and the use of appropriate optical equipment to correct the scattering effect of the clouding on the light beam being measured.

Für die quantitative Erfassung von Gewebsproteinen stehen heute im wesentlichen drei Methoden zur Verfügung: die Bestimmung nach Kjeldahl, die Biuretmethode (1) und deren Modifikation nach Lowry (2) unter Verwendung von Folin-Ciocalteus Phenolreagenz. Von diesen Verfahren werden vor allem die zwei letztgenannten Methoden für die Eiweißbestimmung in Leberhomogenaten angewendet (3-8). Vergleicht man jedoch die. verschiedenen Literaturangaben über den Pro. teingehalt normaler Lebern (z. B. bei der Ratte), so findet man Schwankungen im Bereich von $100-300 \mathrm{mg} / \mathrm{g}$ Leber. Da unseres Erachtens die auftretenden Differenzen nicht allein auf unterschiedliche Aufarbeitungsmethoden zurückgefuihrt werden können, haben wir uns ausfuihrlich mit diesen beiden photometrischen Bestimmungen befaßt.

In der vorliegenden Studie wird an Rattenleberhomogenaten der Einfluß von Fett auf die Proteinbestimmung dargestellt. Gleichzeitig wird nach Möglichkeiten zur Ausschaltung dieser Störung gesucht.

\section{Material und Methoden}

\section{Methoden}

Folgende Methoden kamen bei den Untersuchungen zur Anwendung:

Gesamteiweißbestimmung nach Lowry (2) mit Ciocalteu-Phenolreagenz und nach Weichselbaum (1) mit Biuretreagenz. Lebertrockengewicht und Sedimentsbestimmungen von zentrifugierten Homogenaten wurden nach Lyophilisation gravimetrisch ermittelt.

\section{Apparaturen}

Blutzentrifuge (MSE), Ultraschallgerät (MSE), Photometer (Eppendorf), Vibrator (Vortex, Fa. Bender und Hobein, Zürich), Polytron-Homogenisator (Kinematica, Luzern), Lyophilisator GT-2 (Leybold Heraeus), Pye Unicam SP-1800 UltraviolettSpektrophotometer mit sekundärer Meßposition für Trübungsmessungen.

\section{Reagenzien}

Kupfer[II]-sulfat (Pentahydrat) p.a. Fluka (Nr. 61240) Buchs; Folin-Ciocalteu-Phenolreagenz (Art. Nr. 9001), Natriumdesoxycholat p.a. (Art. Nr. 6504), Chloroform p.a. (Art. Nr. 2445) von Merck, Darmstadt; Brij-35, Levor IV und Aerosol 22 von Technicon Genf; Sterox SE Merz und Dade, Bern, Eiweißstandard: Monitrol I, Merz u. Dade, Bern; Versatol, General Diagnostics (Cosmopharm Zürich); Kaliumnatriumtartrat (Tetrahydrat) Art. 8087 Merck.

Herstellung der Lösungen für Eiweißbestimmungen Eiweißbestimmung nach Lowry

1. $2 \mathrm{~g}$ Natriumcarbonat $\left(\mathrm{Na}_{2} \mathrm{CO}_{3}\right)$ in $1000 \mathrm{ml} \mathrm{0,1} \mathrm{mol} / 1$ Natronlauge

2. $0,5 \mathrm{~g}$ Kupfersulfat $\left(\mathrm{CuSO}_{4} \cdot 5 \mathrm{H}_{2} \mathrm{O}\right)$ in $1000 \mathrm{ml}$ Natriumkaliumtartratlösung* $\left(\mathrm{C}_{4} \mathrm{H}_{4} \mathrm{O}_{6} \mathrm{KNa} \cdot 4 \mathrm{H}_{2} \mathrm{O}: 1 \mathrm{~g} / 100 \mathrm{ml}\right.$ dest. Wasser $)$

3. alkalische Kupferlösung

$50 \mathrm{ml}$ Reagenz 1 und $1 \mathrm{ml}$ Reagenz 2 mischen und 24 Stunden stehen lassen.

4. Folin-Ciocalteus Phenolreagenz (Merck) mit gleichem Volumen dest. Wasser verdünnen.

5. alkalische Tartratlösung (für Leerwertsbest immung) $50 \mathrm{ml}$ Natriumcarbonatlösung (1) und $1 \mathrm{ml}$ Natriumkaliumtartratlösung* $\left(\mathrm{C}_{4} \mathrm{H}_{4} \mathrm{O}_{6} \mathrm{KNa} \cdot 4 \mathrm{H}_{2} \mathrm{O}: 1 \mathrm{~g} / 1000 \mathrm{ml}\right.$ dest. Wasser) mischen.

Eiweißbestimmung nach Weichselbaum

1. Biuretreagenz

Kaliumnatriumtartrat $\left(\mathrm{C}_{4} \mathrm{H}_{4} \mathrm{O}_{6} \mathrm{KNa} \cdot 4 \mathrm{H}_{2} \mathrm{O}\right)$

Natronlauge $(0,2 \mathrm{~mol} / \mathrm{l})$ 
Kupfersulfat $\left(\mathrm{CuSO}_{4} \cdot 5 \mathrm{H}_{2} \mathrm{O}\right)$

Kaliumjodid

2. alkalische Tartratlösung (für Leerwertsbestimmung)

Kaliumnatriumtartrat $\left(\mathrm{C}_{4} \mathrm{H}_{4} \mathrm{O}_{6} \mathrm{KNa} \cdot 4 \mathrm{H}_{2} \mathrm{O}\right)$

Natronlauge $(0,2 \mathrm{~mol} / \mathrm{l})$

$3 \mathrm{~g}$

$5 \mathrm{~g}$

$9 \mathrm{~g}$ $1000 \mathrm{ml}$

Arbeitsschema

Biuret-Methode

\begin{tabular}{|c|c|c|c|c|}
\hline & $\begin{array}{l}\text { Analyse } \\
\text { Weg I } \\
\text { ohne Na- } \\
\text { Desoxy- } \\
\text { cholat }\end{array}$ & $\begin{array}{l}\text { Weg II } \\
\text { mit Na- } \\
\text { Desoxy- } \\
\text { cholat }\end{array}$ & $\begin{array}{l}\text { Analysenl } \\
\text { Weg I } \\
\text { ohne Na- } \\
\text { Desoxy- } \\
\text { cholat }\end{array}$ & $\begin{array}{l}\text { leerwert } \\
\text { Weg II } \\
\text { mit Na- } \\
\text { Desoxy- } \\
\text { cholat }\end{array}$ \\
\hline $\begin{array}{l}\text { Leberhomogenat } \\
(10 \%) \\
\text { Na-Desoxycholat*** }\end{array}$ & $\begin{array}{l}\mu 1 \\
20 \\
-.-\end{array}$ & $\begin{array}{l}\mu l \\
20 \\
1000\end{array}$ & $\begin{array}{l}\mu 1 \\
20 \\
-\end{array}$ & $\begin{array}{l}\mu \mathrm{l} \\
20 \\
1000\end{array}$ \\
\hline \multicolumn{5}{|c|}{15 Minuten bei $37^{\circ} \mathrm{C}$ bzw. $\left(45^{\circ}\right) * *$ für Weg II } \\
\hline $\begin{array}{l}\text { Biuretlösung } \\
\text { Alkalische } \\
\text { Tartratlösung }\end{array}$ & $\begin{array}{r}1000 \\
-\end{array}$ & $\begin{array}{l}1000 \\
-\end{array}$ & $\begin{array}{l}- \\
1000\end{array}$ & $\begin{array}{l}- \\
1000\end{array}$ \\
\hline
\end{tabular}

Nach 30 Minuten bei $37^{\circ}$ Extinktionen bei $546 \mathrm{~nm}$ messen gegen Reagenzienleerwert (RL)

Standard*: Versatol 2,$5 ; 5 ; 10 ; 20 \mu l$

Standardfehler

der Methode

$1,3 \% \quad 1,1 \%$

* Jedem Analysenweg entsprechend wird ein Standard verwendet.

** Inkubation bei $45^{\circ}$ führt $\mathrm{zu}$ besseren Resultaten

*** 20 prozentige wäßrige Lösung

Lowry-Methode

\begin{tabular}{lllll}
\hline & $\begin{array}{l}\text { Analyse } \\
\text { Weg I }\end{array}$ & Weg II & \multicolumn{2}{l}{$\begin{array}{l}\text { Analysenleerwert } \\
\text { Weg I }\end{array}$} \\
& $\begin{array}{l}\text { Weg II } \\
\text { ohne Na- mit Na- } \\
\text { Desoxy- } \\
\text { cholat }\end{array}$ & $\begin{array}{l}\text { Desoxy- } \\
\text { cholat }\end{array}$ & $\begin{array}{l}\text { ohne Na- mit Na- } \\
\text { Desoxy- } \\
\text { cholat }\end{array}$ & $\begin{array}{l}\text { Desoxy- } \\
\text { cholat }\end{array}$ \\
\hline $\begin{array}{l}\text { Leberhomogenat } \\
(1 \%)\end{array}$ & $\mu 1$ & $\mu l$ & $\mu l$ & $\mu l$ \\
$\begin{array}{l}\text { Na-Desoxycholat } \\
(20 \%)\end{array}$ & 20 & 20 & 20 & 20 \\
\hline
\end{tabular}

15 Minuten bei $37^{\circ} \mathrm{C}$

\begin{tabular}{|c|c|c|c|c|}
\hline \multirow{2}{*}{$\begin{array}{l}\text { Alkalische } \\
\text { Kupferlösung } \\
\text { Alkalische } \\
\text { Tartratlösung }\end{array}$} & 1000 & 1000 & & \\
\hline & - & - & 1050 & 1050 \\
\hline \multicolumn{2}{|c|}{10 Minuten stehen lassen } & & & \\
\hline \multicolumn{2}{|c|}{$\begin{array}{l}\text { Folin-Reagenz } / \mathrm{H}_{2} \mathrm{O} 50 \\
(1: 1)\end{array}$} & 50 & - & - \\
\hline
\end{tabular}

Nach 30 Minuten bei $578 \mathrm{~nm}$ (bzw. $660 \mathrm{~nm}$ ) messen gegen RL

Standard*: Versatol $(1: 10$ verdünnt $)-2,5 ; 5 ; 10 \mu 1$

\section{Standardfehler}

der Methode

$2,1 \% \quad 1,7 \%$

${ }^{x}$ Hergestellt aus 10 prozentigem Homogenat durch verdünnen mit Phosphatpuffer $(0,1 \mathrm{~mol} / 1, \mathrm{pH} 7 ; 4)$

\section{Gewebsaufbereitung:}

Jungen Wistar-Ratten ( 8 Wochen) wurden nach Tötung mit $\mathrm{CO}_{2}$ Gas die Lebern sowie Peritonealfett entnommen.

Das peritoneale Fettgewebe wurde anschließend in Chloroform homogenisiert (Polytron), die nicht löslichen Anteile abfiltriert und das Lösungsmittel im Rotationsverdampfer entfernt. Die Lebern wurden in physiologischer NaCl-Lösung mit der Schere zerkleinert, die Teilstücke über Gaze filtriert, mit destilliertem Wasser gewaschen und mit Gaze abgetrocknet. Dann wurde ein Homogenat in $0,1 \mathrm{~mol} / 1$ Phosphatpuffer $(\mathrm{pH} \mathrm{7,4)}$ hergestellt $(1 \mathrm{~g}$ Leber $+9 \mathrm{ml}$ Puffer) und dasselbe 20 Minuten bei $4000 \mathrm{~g}$ zentrifugiert. Der Überstand wurde in 9 Portionen aufgeteilt und diese mit steigenden Mengen Peritonealfett versetzt $(10,20,30,40,50,60,80,160 \mathrm{mg} \mathrm{Fett} / \mathrm{g}$ Leber $)$.

\section{Eiweißbestimmung}

Alle Proteinbestimmungen wurden nach dem voran beschriebenen Arbeitsschema durchgefuhrt, mit und ohne Abzug des Analysenleerwertes. Die in den Tabellen aufgefürten Resultate sind, wenn nicht anders vermerkt, die Mittelwerte der im gleichen Leberhomogenat durchgefürten Bestimmungen. Diese Ergebnisse konnten in Parallelversuchen zusätzlich bestätigt werden.

\section{Ergebnisse}

Zur Rohbilanzierung der Untersuchungen an der Leber wurde zunächst der Wassergehalt bzw. der Trockenrückstand in der Leber bestimmt. Gleichzeitig wurden Homogenate aus Frischlebern hergestellt und nach Zentrifugation die Gewebsanteile im Sediment ermittelt; diejenigen im Uberstand wurden rechnerisch erfaßt. Tabelle 1 zeigt die aus zehn Rattenlebern ermittelten Ergebnisse.

Tab. 1. Wassergehalt der Rattenleber und Trockenrückstände des Leberhomogenates im Sediment und Zentrifugationsüberstand:

Untersuchte Parameter $\begin{gathered}\text { Mittlere Gehaltsangaben aus } 10 \\ \text { verschịedenen Bestimmungen }\end{gathered}$

$$
\overline{\mathrm{x}} \pm \mathrm{s} \text { (mg/g Leber) }
$$

Wassergehalt der Leber

$725 \pm 14$

(Lyophilisationsverfahren)

Trockenrückstand des Sedi-

mentes (nach Zentrifugation)

$$
34 \pm 0,3
$$

Festanteile im Überstand

des Zentrifugates (errechnet)

$241 \pm 0,3$

Diese Bilanzuntersuchung weist nach Abzug des Wassergehaltes in der Rattenleber $275 \mathrm{mg} / \mathrm{g}$ Frischgewicht Festbestandteile auf, die homogenisierbar sind. Zieht man davon die zentrifugierbaren Gewebspartikel ab, so verbleiben für den Analysengang im Überstand günstigenfalls etwa $24 \%$ bestimmbare Gewichtsanteile. Wenn man dann berücksichtigt, daß die Leber auch noch Glykogen, Lipide, anorganische und sonstige organische Bestandteile enthält, wird man in der Annahme bestärkt, daß der in Homogenaten bzw. in Zentrifugaten ermittelte Proteingehalt normaler Rattenlebern unter $200 \mathrm{mg} / \mathrm{g}$ Frischgewicht liegt und Analysenwerte, die darüber liegen, Artefakten zuzuschreiben sind. Nach Angaben von Sodeman (9) sind beispielsweise in der menschli- 
chen Normalleber etwa 3-6\% des Feuchtgewichtes fettähnliche Substanzen. Bei Leberverfettung können (bezogen auf Totallipide) Lipidanteile bis zu $40 \%$ gefunden werden. Remmer (10) beschreibt bei chemisch induzierten Fettlebern (Ratte) auf der Basis von Triglyceridbestimmungen Fettanteile bis zu $5 \%$ des Feuchtgewichtes.

Auf Grund dieser Angaben wurde versucht, durch Zugabe von peritonealem Rattenfett zu den entsprechenden Leberhomogenaten ähnliche Verhältnisse zu simulieren, um zu überprüfen, wie stark die Meßwerte von Eiweißbestimmungen durch die Anwesenheit verschiedener Fettmengen beeinflußt werden.

$\mathrm{Zu}$ diesem $\mathrm{Zwecke}$ wurden in Rattenleberhomogenaten mit konstantem Protein- und steigendem Fettgehalt Eiweißbestimmungen durch Messung am Eppendorf Photometer nach dem angegebenen Arbeitsschema unter verschiedenen Gesichtspunkten durchgeführt und die Ergebnisse in Tabelle 2 zusammengestellt.

1. Die Proteinbestimmung mit Biuret ergibt ohne Leerwertsabzug und ohne Hilfszusätze mit steigender Fettkonzentration eine Zunahme des methodischen
Fehlers (Trübungseffekt), der im Extremfall (bei $16 \%$ Fettzusatz) bis zu $1000 \%$ betragen kann. Bei der Lowry-Methode führen steigende Fettgehalte ebenfalls zu einer Beeinflussung der Resultate (Fehler bis $43 \%$ bei $16 \%$ Fettzusatz). Vergleichend betrachtet, erweist sich unter diesen Meßbedingungen die Biuretmethode fur Gewebseiweißbestimmung als nicht sehr geeignet; dagegen liefert die Lowry-Methode bis zu einem Fettzusatz von $4 \%$ brauchbare Werte, vorausgesetzt man akzeptiert einen Analysenfehler bis zu $10 \%$.

2. Versucht man den Trübungseffekt des Fettes durch Leerwertsabzug auszuschalten, ergeben sich bei der Folin-Reaktion durchaus brauchbare Werte, obwohl auch hier bei höheren Fettkonzentrationen (8-16\% Fettzusatz) eine leichte Abnahme der Proteinwerte sichtbar wird. Bei der Bestimmung mit Biuretreagenz versagt diese Differenzmessung vollständig. Die schlecht reproduzierbaren Proteinwerte fallen zu niedrig aus, und sie werden zudem mit steigender Fettkonzentration immer kleiner (Tyndall-Effekt).

3. Auf Grund obiger Resultate wurde versucht, die

Fettrübung mit Detergenzien auszuschalten (B rij- 35,

Tab. 2. Total-Protein Bestimmung an Rattenleberhomogenaten mit konstantem Proteingehalt in Abhängigkeit zur zugesetzten Menge an peritonealem Rattenfett (Mittelwerte aus je drei Analysen des gleichen Homogenates). ( $\overline{\mathrm{X}} \pm \Delta \mathrm{X}$ : Mittlerer Meßwert \pm Standardabweichung).

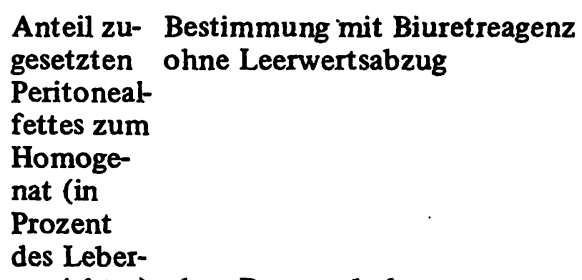

\begin{tabular}{|c|c|c|c|c|c|c|c|c|}
\hline \multirow[b]{2}{*}{$\begin{array}{r}0 \\
1 \\
2 \\
3 \\
4 \\
5 \\
6 \\
8 \\
16\end{array}$} & \multirow{2}{*}{$\begin{array}{l}\begin{array}{l}\text { Protein in } \mathrm{mg} / \mathrm{g} \\
\text { Leber } \\
\overline{\mathbf{x}} \pm \Delta \mathrm{X} \\
180 \pm 5 \\
238 \pm 3 \\
351 \pm 6 \\
574 \pm 8 \\
594 \pm 11 \\
762 \pm 7 \\
906 \pm 7 \\
1202 \pm 9 \\
2058 \pm 36\end{array}\end{array}$} & $\begin{array}{l}\begin{array}{l}\text { Abweichung } \\
\text { der Protein- } \\
\text { werte in \% }\end{array} \\
-\end{array}$ & $\begin{array}{l}\begin{array}{l}\text { Protein in } \mathrm{mg} / \mathrm{g} \\
\text { Leber } \\
\overline{\mathrm{X}} \pm \Delta \mathrm{X}\end{array} \\
149 \pm 4 \\
145 \pm 2\end{array}$ & $\begin{array}{l}\begin{array}{l}\text { Abweichung } \\
\text { der Protein- } \\
\text { werte in } \%\end{array} \\
-\end{array}$ & $\begin{array}{l}\text { Protein in } \mathrm{mg} / \mathrm{g} \\
\text { Leber } \\
\overline{\mathrm{X}} \pm \Delta \mathrm{X}\end{array}$ & $\begin{array}{l}\begin{array}{l}\text { Abweichung } \\
\text { der Protein- } \\
\text { werte in \% }\end{array} \\
-\overline{1}\end{array}$ & $\begin{array}{l}\text { Protein in } \mathrm{mg} / \mathrm{g} \\
\text { Leber } \\
\overline{\mathbf{x}} \pm \Delta \mathrm{X}\end{array}$ & \multirow{2}{*}{$\begin{array}{l}\begin{array}{l}\text { Abweichung } \\
\text { der Protein- } \\
\text { werte in \% }\end{array} \\
- \\
-1 \\
-2 \\
-4 \\
0 \\
-5 \\
+1 \\
-2 \\
+3\end{array}$} \\
\hline & & $\begin{array}{r}- \\
+\quad 32 \\
+95 \\
+219 \\
+230 \\
+323 \\
+403 \\
+568 \\
+1042\end{array}$ & $\begin{array}{l}149 \pm 4 \\
145 \pm 2 \\
150 \pm 2 \\
166 \pm 2 \\
161 \pm 3 \\
175 \pm 5 \\
179 \pm 1 \\
204 \pm 4 \\
300 \pm 6\end{array}$ & $\begin{array}{rr} & - \\
-\quad 3 \\
+\quad 1 \\
+\quad 11 \\
+\quad 8 \\
+\quad 17 \\
+20 \\
+37 \\
+101\end{array}$ & $\begin{array}{l}141 \pm 4 \\
143 \pm 2 \\
148 \pm 4 \\
153 \pm 2 \\
157 \pm 3 \\
163 \pm 6 \\
170 \pm 4 \\
176 \pm 5 \\
202 \pm 10\end{array}$ & $\begin{array}{l} \\
+1 \\
+5 \\
+8 \\
+11 \\
+16 \\
+21 \\
+25 \\
+43\end{array}$ & $\begin{array}{l}142 \pm 5 \\
141 \pm 8 \\
140 \pm 8 \\
137 \pm 3 \\
142 \pm 7 \\
135 \pm 2 \\
143 \pm 2 \\
139 \pm 6 \\
146 \pm 4\end{array}$ & \\
\hline & \multicolumn{4}{|c|}{ mit Leerwertsabzug } & \multicolumn{4}{|c|}{ mit Leerwertsabzug } \\
\hline $\begin{array}{r}0 \\
1 \\
2 \\
3 \\
4 \\
5 \\
6 \\
8 \\
16\end{array}$ & $\begin{array}{l}96 \pm 9 \\
86 \pm 0 \\
70 \pm 0 \\
61 \pm 0 \\
70 \pm 3 \\
67 \pm 4 \\
67 \pm 4 \\
67 \pm 4 \\
35 \pm 13\end{array}$ & $\begin{array}{l}- \\
-\quad 11 \\
-\quad 28 \\
-\quad 37 \\
-\quad 28 \\
-\quad 30 \\
-\quad 30 \\
-\quad 30 \\
-\quad 64\end{array}$ & $\begin{array}{r}150 \pm 2 \\
150 \pm 1 \\
145 \pm 4 \\
145 \pm 3 \\
130 \pm 2 \\
120 \pm 4 \\
110 \pm 7 \\
110 \pm 5 \\
75 \pm 9\end{array}$ & $\begin{array}{r}- \\
0 \\
-\quad 3 \\
-\quad 3 \\
-14 \\
-21 \\
-27 \\
-27 \\
-50\end{array}$ & $\begin{array}{l}146 \pm 2 \\
147 \pm 0 \\
147 \pm 4 \\
142 \pm 2 \\
147 \pm 4 \\
147 \pm 4 \\
143 \pm 4 \\
139 \pm 4 \\
135 \pm 4\end{array}$ & $\begin{array}{r}- \\
+1 \\
+1 \\
-3 \\
+1 \\
+1 \\
-2 \\
-5 \\
-8\end{array}$ & $\begin{array}{l}141 \pm 0 \\
143 \pm 2 \\
147 \pm 4 \\
144 \pm 2 \\
141 \pm 0 \\
139 \pm 6 \\
142 \pm 5 \\
145 \pm 4 \\
136 \pm 4\end{array}$ & $\begin{array}{r}- \\
+\quad 1 \\
+4 \\
+\quad 2 \\
0 \\
-1 \\
+1 \\
+3 \\
-4\end{array}$ \\
\hline
\end{tabular}

mit Desoxycholat-Na (20\%) 
Levor IV, Sterox SE, Aerosol und Natriumdesoxycholat). Hierbei zeigte sich nur Natriumdesoxycholat bei beiden Methoden zur Unterdrückung der Fettrübung geeignet. In Anwesenheit von diesem Detergenz liefert die Biuretmethode bei Fettanteilen bis zu $4 \%$ brauchbare Meßresultate. Höhere Fettkonzentrationen ergeben ohne Leerwertsabzug höhere Proteinwerte (max. bis $100 \%$ bei $16 \%$ Fettzusatz). Leerwertsabzug dagegen fuihrt bei Fettkonzentrationen über $5 \%$ zu niederen Resultaten. Bei der LowryMethode finden sich jedoch nach Zusatz von Desoxycholat (mit und ohne Leerwertsabzug) bei verschiedenen Fettkonzentrationen konstante Proteinwerte.

4. Untersuchungen mit weiteren Hilfsstoffen (z. B.

Harnstoff, Propandiol-1,2), die in der Literatur z. T. für Proteinbestimmungen im trüben Serum empfohlen werden $(11,12)$, führen bei der Bestimmung mit Biuretreagenz in Homogenaten nicht zu den erwünschten Resultaten (siehe Tab. 3). Auch die Entfärbung der Biuret-Reaktion mit KCN und Abzug des dadurch erhaltenen Probenleerwertes ergibt keine brauchbaren Ergebnisse (Überkorrektur). Extraktion des Fettes mit organischen Lösungsmitteln und an- schließende Bestimmung des Proteins mit Biuretbzw. Folin-Reagenz führt natürlich unter den gegebenen Bedingungen zur Beseitigung des Fett-Trübungseinflusses.

Weiterhin fiel uns bei den oft wiederholten Untersuchungen auf, daß der-Trübungsfehler bei der Bestimmung mit Biuretreagenz je nach Aufarbeitungsverfahren und Dispergierung zum Teil beträchtlich variieren kann (Fettschichtung).

5. Zusätzlich wurde der Einfluß verschiedener Fettmengen auf verschiedene Proteinmengen untersucht. Dabei zeigte sich, daß beides, Eiweiß- und Fettgehalt, für die Meßfehlergröße bei der Bestimmung mit Biuretreagenz verantwortlich sind (Tab. 4). Bei einem Fettzusatz bis $8 \%$ ist der Trübungsfehler umso größer, je kleiner der Eiweißgehalt ist; bei höheren Fettanteilen finden wir ohne Berücksichtigung besonderer Verșuchsvorkehrungen infolge gesteigerter Fettschichtung eine Verminderung in der Zunahme des Trübungseffektes.

6. Die vorwiegend auf den Tyndall-Effekt zurückzuführenden schlechten Ergebnisse bei der Bestimmung mit Biuretreagenz mit Leerwertśabżug veranlaßten

Tab. 3. Total-Protein Bestimmung an Rattenleberhomogenaten* mit konstantem Proteingehalt in Abhängigkeit zur zugesetzten Menge an peritonealem Rattenfett (Mittelwerte aus je drei Analysen des gleichen Homogenates). Messung in Anwesenheit verschiedener Hilfsstoffe.

$(\overline{\mathrm{x}} \pm \Delta \mathrm{X}:$ Mittlerer Meßwert \pm Standardabweichung) .

\begin{tabular}{|c|c|c|c|c|c|c|c|c|}
\hline \multirow{3}{*}{$\begin{array}{l}\text { Anteil zu- } \\
\text { gesetzten } \\
\text { Peritone- } \\
\text { alfettes } \\
\text { zum Ho- } \\
\text { mogenat } \\
\text { (in \% des } \\
\text { Leberge- } \\
\text { wichtes) }\end{array}$} & \multicolumn{8}{|c|}{ Bestimmung mit Biuretreagenz (ohne Leerwertsabzug) } \\
\hline & \multicolumn{2}{|l|}{ ohne Zusatz } & \multicolumn{2}{|l|}{ mit Harnstoff } & \multicolumn{2}{|c|}{ mit Propandiol-1,2 } & \multicolumn{2}{|c|}{ mit Desoxycholat-Na (20 \%) } \\
\hline & $\begin{array}{l}\text { Protein in } \mathrm{mg} / \mathrm{g} \\
\text { Leber } \\
\overline{\mathbf{x}} \pm \Delta \mathrm{X}\end{array}$ & $\begin{array}{l}\text { Abweichung } \\
\text { der Protein- } \\
\text { werte in } \%\end{array}$ & $\begin{array}{l}\text { Protein in } \mathrm{mg} / \mathrm{g} \\
\text { Leber } \\
\overline{\mathbf{x}} \pm \Delta \mathbf{X}\end{array}$ & $\begin{array}{l}\text { Abweichung } \\
\text { der Protein- } \\
\text { werte in \% }\end{array}$ & $\begin{array}{l}\text { Protein in } \mathrm{mg} / \mathrm{g} \\
\text { Leber } \\
\overline{\mathbf{X}} \pm \Delta \mathbf{X}\end{array}$ & $\begin{array}{l}\text { Abweichung } \\
\text { der Protein- } \\
\text { werte in \% }\end{array}$ & $\begin{array}{l}\text { Protein in } \mathrm{mg} / \mathrm{g} \\
\text { Leber } \\
\overline{\mathbf{x}} \pm \Delta \mathbf{X}\end{array}$ & $\begin{array}{l}\text { Abweichung } \\
\text { der Protein- } \\
\text { werte in } \%\end{array}$ \\
\hline $\begin{array}{r}0 \\
4 \\
8 \\
15 \\
30\end{array}$ & $\begin{array}{r}177 \pm 0 \\
407 \pm 12 \\
782 \pm 8 \\
1975 \pm 60 \\
2221 \pm 23\end{array}$ & $\begin{array}{r}- \\
+\quad 130 \\
+342 \\
+1016 \\
+1155\end{array}$ & $\begin{aligned} 200 & \pm 18 \\
500 & \pm 22 \\
860 & \pm 51 \\
880 & \pm 52 \\
1280 & \pm 32\end{aligned}$ & $\begin{array}{r}- \\
+150 \\
+330 \\
+340 \\
+540\end{array}$ & $\begin{array}{r}180 \pm 7 \\
590 \pm 14 \\
1110 \pm 7 \\
1280 \pm 30 \\
1710 \pm 45\end{array}$ & $\begin{array}{l}+ \\
+228 \\
+516 \\
+611 \\
+850\end{array}$ & $\begin{array}{l}150 \pm 0 \\
165 \pm 0 \\
195 \pm 6 \\
355 \pm 10 \\
560 \pm 0\end{array}$ & $\begin{array}{r}+\overline{10} \\
+\quad 30 \\
+136 \\
+273\end{array}$ \\
\hline
\end{tabular}

* Neu hergestelltes Leberhomogenat.

Tab. 4. Totalproteinbestimmung mit Biuretreagenz in Anwesenheit von verschiedenen Fett- und Eiweißmengen (ohne Leerwertsabzug).

\begin{tabular}{|c|c|c|c|c|c|c|}
\hline $\begin{array}{l}\text { Fettzusatz in \% } \\
\text { des „Leberge- } \\
\text { wichtes“ }\end{array}$ & $\begin{array}{l}\text { Eiweißgehal } \\
\mathrm{mg} / \mathrm{g}\end{array}$ & $\begin{array}{l}\text { Leber" } \\
\text { Fehler } \\
\text { in \% }\end{array}$ & $\mathrm{mg} / \mathrm{g}$ & $\begin{array}{l}\text { Fehler } \\
\text { in \% }\end{array}$ & $\mathrm{mg} / \mathrm{g}$ & $\begin{array}{l}\text { Fehler } \\
\text { in \% }\end{array}$ \\
\hline $\begin{array}{r}0 \\
4 \\
8 \\
15 \\
30 \\
\end{array}$ & $\begin{array}{r}177 \pm 0 \\
407 \pm 12 \\
782 \pm 8 \\
1975 \pm 60 \\
2221 \pm 23\end{array}$ & $\begin{array}{r}- \\
+\quad 130 \\
+342 \\
+1016 \\
+1155\end{array}$ & $\begin{array}{r}127 \pm 0 \\
367 \pm 5 \\
961 \pm 5 \\
1170 \pm 21 \\
1400 \pm 48\end{array}$ & $\begin{array}{r}- \\
+\quad 189 \\
+\quad 656 \\
+821 \\
+1002\end{array}$ & $\begin{array}{r}79 \pm 5 \\
403 \pm 14 \\
639 \pm 28 \\
773 \pm 18 \\
876 \pm 36\end{array}$ & $\begin{array}{r}- \\
+\quad 410 \\
+\quad 709 \\
+\quad 879 \\
+1009\end{array}$ \\
\hline
\end{tabular}

* Hergestellt durch Verdünnen von Leberhomogenat mit Phosphatpuffer. Die Fettmengen wurden dann einzeln zugewogen. 
uns, diese sogenannte Differenzmethode (Biuret) am Pye Unicam SP-1800 mit einer speziellen Einrichtung für Trübungsmessungen $\mathrm{zu}$ wiederholen. Hierbei zeigte sich, daß bei dieser Meßanordnung die Proteinbestimmung mit Desoxycholat brauchbare Werte liefert. Ohne Detergenz sind die Resultate bis $8 \%$ Fettgehalt annehmbar, sie fallen jedoch durchschnittlich etwas niedriger aus (Tab. 5).

Tab. 5. Totalproteinbestimmung mit Biuretreagenz unter Abzug der Leerwerte, gemessen am Unicam-Photometer SP-1800 in sekundärer Meßposition (Mittelwert aus zwei Bestimmungen des gleichen Homogenates).

\begin{tabular}{lllll}
\hline $\begin{array}{l}\text { Anteil zuge- } \\
\text { setzten Peri- } \\
\text { tonealfettes } \\
\text { zum Homo- }\end{array}$ ohne Desoxycholat & \multicolumn{3}{l}{ mit Desoxycholat } \\
genat (in Pro- Protein in \\
$\begin{array}{l}\text { zent des Le- } \\
\text { bergewichtes) }\end{array}$
\end{tabular}

* Neu hergestelltes Leberhomogenat.

$\overline{\mathbf{x}} \pm \Delta \mathrm{X}:$ Mittlerer Meßwert \pm Standardabweichung

\section{Diskussion}

Die Proteinbestimmung in Homogenaten ist noch heute ein analytisches Problem. Es besteht im wesentlichen darin, daß Absorptions- bzw. Transmissionsmessungen, die dem Beer-Lambert'schen-Gesetz unterliegen, im trüben Milieu vorgenommen werden müssen und dadurch vom sogenannten Tyndall-Effekt (Streulichteffekt) überlagert werden. Aus diesem Grunde können z. T. bei der photometrischen Messung von Gewebsproteinen (ohne Leerwertsabzug und ohne Hilfszusätze) höhere Resultate, bei Differenzmessungen mit Spektrophotographen ohne spezielle Einrichtung zur Ausschaltung bzw.
Unterdrückung der Streustrahlung niedere Werte resultieren. Wir haben in der vorliegenden Arbeit am Beispiel der Fettrübung diese Problematik behandelt; hierbei ergaben sich zwei Möglichkeiten zur direkten Unterdrükkung der Fehlerquellen:

1. Beseitigung der Trübungsursache durch Detergenzzusatz (Desoxycholat).

2. Verwendung von Spektralphotometern mit Meßeinrichtungen, die geeignet sind, die Streustrahlung zu unterdrücken (evtl. Einbau von küvettennahen „Streustrahl-Sammellinsen“" in gewöhnliche Routinegeräte).

Beim Vergleich der beiden photometrischen Methoden zeigt die Reaktion nach Folin-Ciocalteu bei der Gewebsproteinbestimmung unter herkömmlichen Bedingungen gegenüber der reinen Biuretreaktion einen gewissen Vorteil. Dieser ist darauf zurückzuführen, daß der auf dem Folin-Reagenz basierende zusätzliche Reduktionsschritt (Reduktion von Heteropolysäuren zu blauem Farbstoff) die Methode etwa $10 \mathrm{mal}$ empfindlicher macht. Dadurch wird natürlich für die Proteinbestimmung die notwendige Menge an Homogenat und der Einfluß des Trübungseffektes entsprechend vermindert. Allerdings müssen wir bei Verwendung von Folin-Reagenz eine geringere Spezifität durch die bekannten Interferenzen mit Substanzen wie z. B. Kohlenhydrate, Purine und gewisse Arzneimittel in Kauf nehmen (13-15).

Die viel spezifischere Biuretreaktion sollte bei der Gewebsproteinbestimmung nur bei Vorhandensein geeigneter Spektrophotometer mit spezieller Anordnung für Trübungsmessungen und unter Abzug des Analysenleerwertes vorgenommen werden. Andernfalls ist die Lowry-Methode vorzuziehen, wie die voran beschriebenen Resultate zeigen.

Der Gewebsproteingehalt wird heute vielfach als Bezugsgröße für andere Gewebsparameter verwendet, und Unklarheiten in der Proteinbestimmung können daher sehr leicht auf Grund der beschriebenen Artefakte zu falschen Schlußfolgerungen verleiten. Wir hoffen, mit der vorliegenden Arbeit zur Klärung dieses analytischen Problems beitragen zu können.

\section{Danksagung}

Für die Mithilfe bei der Durchführung der Bestimmungen möchten wir Frl. A. Schütze und Frl. D. Ulrich herzlich danken.

\section{Literatur}

1. Weichselbaum, T. E. (1946), Amer. J. Clin. Pathol. 7, 40.

2. Lowry, O. H., Rosebrough, N. J., Farr, A. L., \& Randa (1951), J. Biol. Chem. 1993, 265-275.

3. Murphy, S. D., \& Malley, S. (1969), Toxicol Appl. Pharmacol. 15, 117-130.

4. Dewhurst, F., \& Kitchen, D. A. (1973), Biochem. Pharmacol, $22,561-566$
5. Jori, A., Pescador, R., \& Pugliatti, C. (1971), Biochem. Pharmacol., 20, 2695-2701.

6. Argyris, T. S., \& Magnus, D. R. (1968), Develop. Biol. 17, 187-201.

7. Leighton, F., Poole, B., Beaufay, H., Baudhuin, P., Coffey, J. W., Fowler, S., \& De Duve, Ch. (1968), J. Cell. Biol., $37,482-513$. 


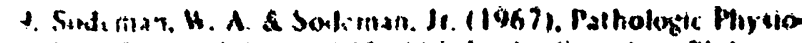

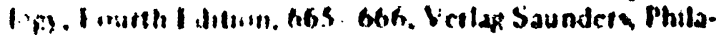
Airpivas.

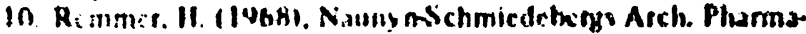
hill. Tir Pathel 26n, 90.

11 Risllinit. R.. (1970). Klineschi Chemie, Theoric und

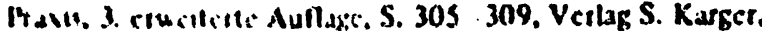
Bail.
12 I.uttermanin, S., \& Rollint M. H. (1973). Anal Biochem. $31.443-447$.

13. Bonitati, J., E.lluot, W. B., Milet P. G. (1969). Anal 810 chem. 31. 399 - 404.

14. Bucter, F.N. (1972), Anal. Blochem. 50, 500-502.

15. Parhley, D. H. Schustct, G. S., Palmcr, P., \& Sharawy (1973). Clun. Chem. 19.263 265.

Dr. rer. nat. Alfons Metz. Sandoz AC,

CH-4002 Bascl (Schweia) 